\title{
Infected dentigerous cyst due to traumatic injury in impacted of mandible canine: a case report
}

\author{
Idawati Muhajir, ${ }^{1 *}$ Seto Adiantoro ${ }^{2}$, Andri Hardianto, ${ }^{1}$ Kiki A. Rizki $^{1}$
}

\begin{abstract}
Objective: This case study is a case of infected dentigerous cyst involving all erupted incisor teeth and impacted mandible canine. Diagnosis of results from clinical examination, radiographic and histopathologic.

Methods: The initial treatment was antibiotic therapy for 2 week, followed with cyst enucleation, extracted of the all fourth incisors and odontectomy lower left canine, lower premolar and upper right molar

through intraoral approach under general anesthesia.

Results: The evaluation of 2 weeks post surgery treatment showed the healing was good and the chin region showed no sign of inflammation, the color of the tissue was similar to its surrounding.

Conclusion: Last sentence of abstract should be a conclusion or further treatment plan for the case. Histological examination and medical history should be placed before the treatment procedure.
\end{abstract}

${ }^{1}$ Department of Oral and Maxillofacial Surgery, Faculty of Dentistry, Padjadjaran University, General Hospital Dr. Hasan Sadikin Bandung, Indonesia

${ }^{2}$ Department of Oncology Surgery, Faculty of Dentistry, Padjadjaran University, Bandung, Indonesia

*Correspondence to:

ifulida83.82@gmail.com

Received: 3 November 2018

Revised: 2 March 2019

Accepted: 7 January 2019

Available Online: 1 May 2019

Keywords: Dentigerous cyst, Enucleation, Infection

Cite this Article: Muhajir I, Adiantoro S, Hardiantoro A, Rizki KA. 2019. Infected dentigerous cyst due to traumatic injury in impacted of mandible canine: a case report. Journal of Case Reports in Dental Medicine. 1(1): 14-17. D0l: 10.20956/jcrdm.v1i1.85

\section{Introduction}

Dentigerous cyst is a common oral lesion formed by fluid accumulation between the fully formed tooth crown and the reduced enamel epithelium. It is considered as a developmental abnormality arising due to reduced enamel epithelium around the crown of an unerupted tooth. While a normal follicular space is 3 to 4 $\mathrm{mm}$, a dentigerous cyst can be suspected when the space is more than $5 \mathrm{~mm} \cdot .^{1-3}$ The cyst arises from the separation of follicle from the crown of an unerupted tooth. Though it may involve any tooth, mandibular third molars are most commonly affected. ${ }^{4}$ They are frequently noted as incidental findings on the radiographs because majority of these cysts are asymptomatic, unless they become secondarily infected. Radiograph shows unilocular, radiolucent lesion characterized by well-defined sclerotic margins and associated with the crown of an unerupted tooth., ${ }^{2,5}$

Clinical symptoms that appear to be slow, painless, hard palpation suggest a cortical expansion. If there is pain and swelling which grow quickly indicates the presence of inflammation. ${ }^{4,6}$ If the cyst is aspirated it will obtain a clear yellowish liquid.?

Histological features of cyst wall show that the cyst is covered by reduced enamel epithelium. The findings depend on whether there is an inflammatory component of the cyst. Uninfected cysts have a solid layer of 2-4 layered epitelium composed of primitive ectomesenchyma. This cell layer is slightly cuboid than columnar and looks rete peg. The loose connective tissue stroma is rich in acid muccopolysaccharides. Characteristics of inflamed dentigerus cysts are hyperplastic rete ridge and cyst wall exhibit inflammatory infiltration. The inflammatory cysts and other enlarged cysts such as dentigerus and odontogenic cysts keratosis can be the main cause. The dentoalveolar abscess is a lesion derived from a pyogenic infection of periodontal and bone ligaments due to a direct expansion of acute pulp, acute non periodontitis, or acute exacerbation of granuloma, cysts or chronic abscess. ${ }^{1,4}$

The purpose of this case study is a case of infected dentigerous cyst involving all erupted incisor teeth and impacted mandible canine. Diagnosis of results from clinical examination, radiographic and histopathologic. As well as to given information to clinicians about surgical enucleation of cysts, removal of tooth impaction and treatment for infection of dentigerus cysts infected with swelling at ekstra oral region and intra-oral medical approaches. Patients also agree that the disease is published for the benefit of science by signing informed consent.

\section{Case Report}

The 25 years old male patient reported to Department of Oral and Maxillofacial Surgery Hasan Sadikin Hospital with swelling, pain and 
at chin region. The swelling oftenly appears and persisted for the passed one year. On extra oral examination, there was swelling, pain and redness at chin region. Intra oral examination there was no swelling present, tenderness at all fourth incisors and canines of lower jaw region figure 1 . Aspiration was pus in the mentale region. The radiographic x-ray showed there was radiolucency at apical region of the all fourth incisors and lower canine with $\pm 2.5 \times 2 \mathrm{~cm}$ size. Other findings include impacted of impacted of left lower canine, lower premolars and right uppper molar figure 2. Initial treatment was antibiotic therapy for 2 week, followed with cyst enucleation, extracted of the all fourth incisors and odontectomy of left lower canine, lower premolars and right uppper molar through intraoral approach under general anesthesia. Figure $3 \pm 5$ years ago the patient was history of traumatic injury with lacerated wound at chin region, then the patient was hospitalized in Garut area and performed suturing at lacerated in chin region. The evaluation of 2 weeks post surgery treatment showed the healing was good and the chin region showed no sign of inflammation, the color of the tissue was similar to its surrounding. Histological examination revealed an infected dentigerus cyst. Figure 4 and figure 5.

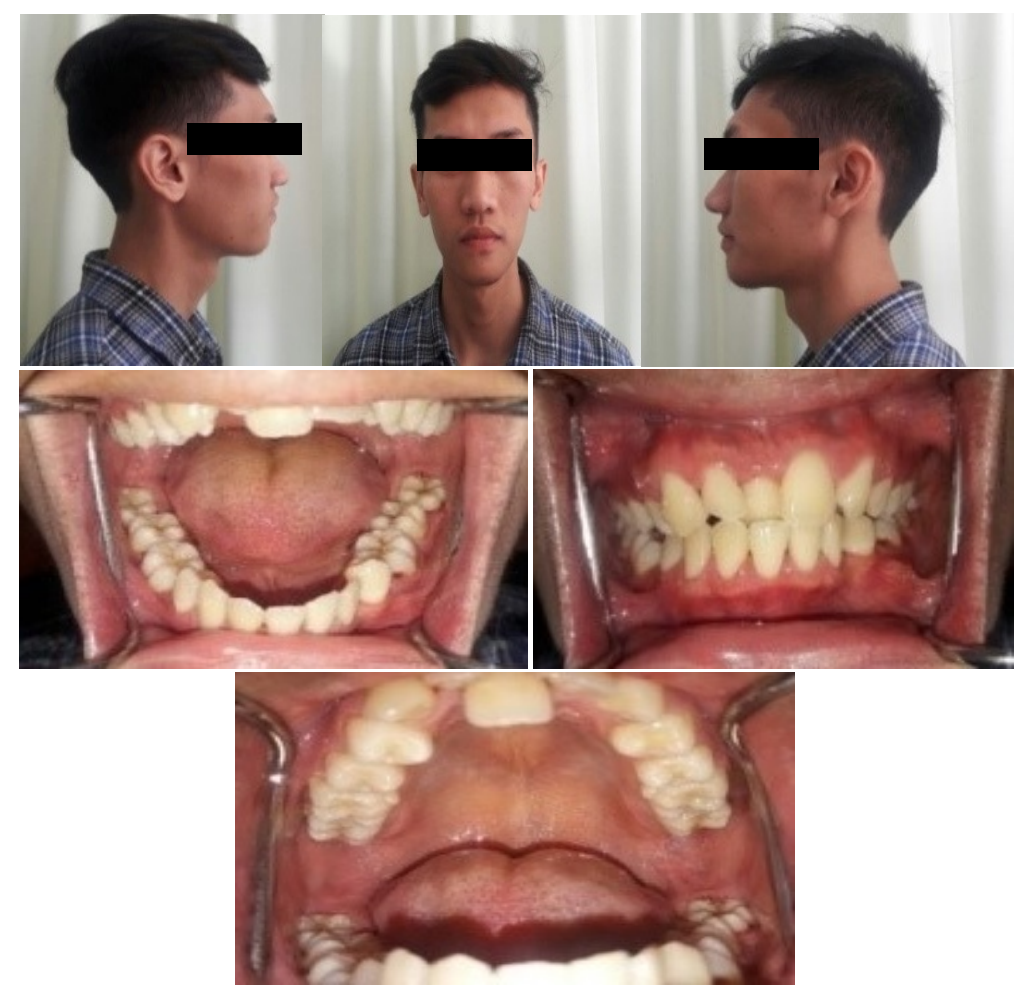

Figure 1 Extra oral and intar oral examination showed no significant findings

\section{Discussion}

Dentigerous cysts are benign odontogenic cysts related with crowns of impacted permanent teeth. These cysts are mostly discovered by routine radiography or by swelling of affected region in the jaw. Their attachment with the cemento-enamel junction of the involved teeth forms the prime basis for their diagnosis. ${ }^{3,8} \mathrm{In}$ order of decreasing frequency, they are associated with mandibular third molars, maxillary canines, mandibular second premolars, maxillary third molars, mandibular first premolar, maxillary second premolar and mandibular canine. . $^{2,5}$,

Radiographically, three variants of dentigerous cysts namely central, lateral and circumferential have been described. These variants invariably demonstrate a unilocular radiolucency with sclerotic borders surrounding the crown of the tooth but as a rule of thumb, un-erupted or partial eruption of the tooth is considered mandatory for diagnosis of dentigerous cyst. A large dentigerous cyst may appear multilocular radiographically owing to the persistence of bone trabeculae within the radiolucency. ${ }^{2,3,8}$

In our case, the cyst was multilocular and associated with the neck of mandibular canine and apical all fourth insisors. The growth of the cyst in the present case cyst had occurred predominantly in the radiks direction allowing the crown to erupt normally. The initial diagnosis of this case is an infected dentigerus cyst on the chin. This diagnosis was based on the clinical appearance of lesions that are slow-growing, pain, swelling and the presence of an extra oral fistle that secretes pus. The radiographic $\mathrm{x}$-ray showed variant of lateral type of dentigerous cyst. The initial cause may be trauma that happened about 5 years ago. there was a wound at chin that bacteria enter the microorganisme between soft tissue and alveolar bone to form an infection at the root tip. This chronic infection extended with radially to alveolar bone. The thin alveolar bone of the cyst wall was easily penetrated by chronic inflammatory infections and causes infection to develop in the cyst. The above finding is extremely rare.

The theory of dentigerous cyst development involves two things: the theory of stimulation and the theory of inflammation. Dentigerous cysts usually appear from the enamel organs after the amelogenesis stage is complete then the excess fluid that causes the separation of enamel from the unerupted teeth. This hyperosmolarity fluid causes the extracellular fluid into the cyst causing the expansion of the cyst to the surrounding tissue. The epithelial layer of the cyst then secretes the factors that activate collagenase and osteoclast 


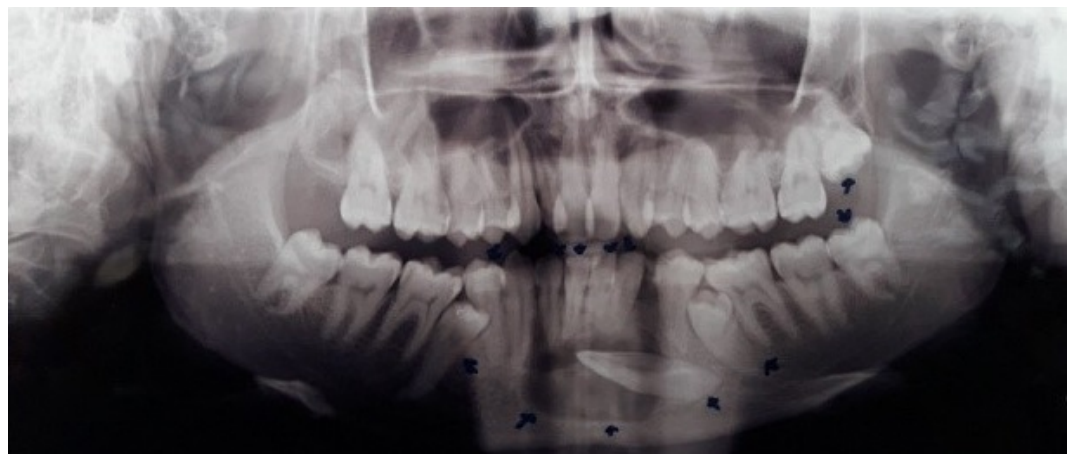

Figure 2 The Panoramic $x$-ray showed an impacted of left lower canine, lower premolars and right uppper molar there was radiolucency at apical region of the all fourth incisors and canine of lower jaw

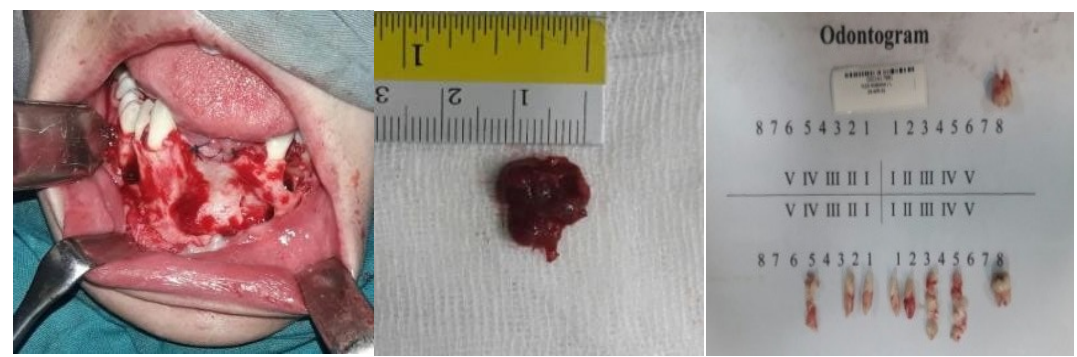

Figure 3 Surgically excised cyst and extraction of teeth

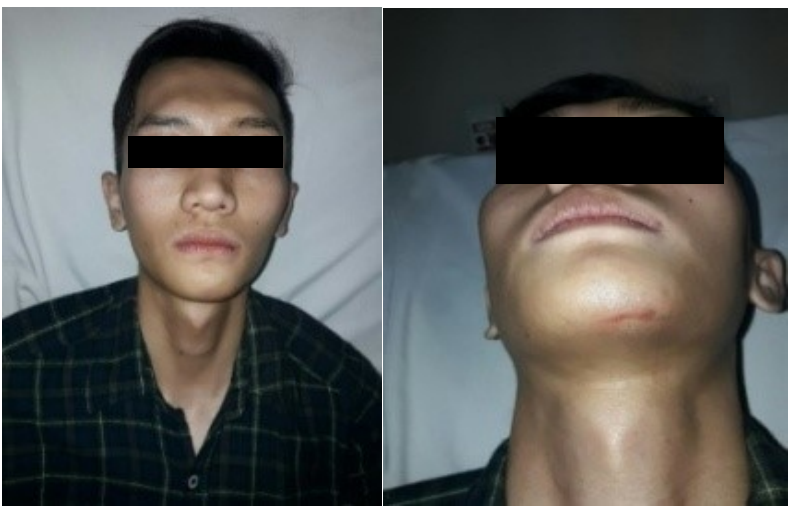

Figure 4 Extra oral examination showed the healing of submentale abscess

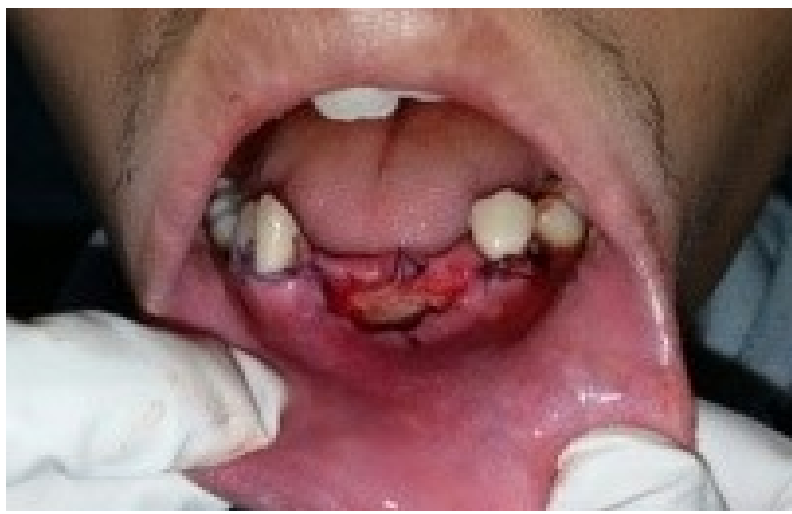

Figure 5 Control post operation day 7 before aff hecting causing bone resorption and the size of the cyst to become larger. This enlarged cyst then envelops the entire root of four mandibular incisors, impacted mandibular canine and attaches to the cementoenamel junction.

Recommended treatment for dentigerous cyst is mostly marsupialization, if the involved tooth might be brought into its normal position in the arch. Enucleation of cyst with removal of the associated tooth is recommended if it shows arrested development or is extensively displaced. In our case, medical therapy, enucleation of the cyst along with the affected teeth was done.

The case was medical therapy with antibiotics for infection healing done 3 weeks before enucleation. The evaluation of 2 weeks showed the healing of submentale abscess. The operation through intra oral approach under genaral anesthesia, cyst was enucleated with removal of the impacted at left maxilllary third molar, left mandible third molar, left mandible canine, bilateral premolar mandible and extraction of fourt insisors mandible. The excised tissue was sent for histopathological examination. The evaluation of 2 weeks post surgery treatment showed healing and tissue color according to approximately, histologic examination results showed an overview of an infected dentigerous cyst.

\section{Conclusion}

The present case we reported a rare case of infected dentigerous cyst due to traumatic injury associated in impacted of mandibular canine and erupted fourth insisors. Treatment is medication with antibiotic, cyst enucleation, odontectomy and extraction of teeth that are involved in the case of an infected dentigerus cyst with intraoral approach. The chronic infection procces in dentigerous cyst could spread slowly forming abscess. The case was successfully treated with no complications post operative.

\section{Aknowledgment}

None.

\section{Conflict of Interest}

The authors report no conflict of interest.

\section{References}

1. Motamedi MHK, Talesh KT. Management of extensive dentigerous cysts. 2005;198: 203-206.

2. Shergill AK, Singh P, Charlotte M. Dentigerous cyst associated with an erupted tooth-an unusual presentation. 2014;2: 100-102. 
3. Andersson L, Kahnberg KE. Oral and maxillofacial surgery: Blackwell Publishing Ltd; 2010. p. 8.

4. Veera SD, Padanad G. Dentigerous cyst with recurrent maxillary sinusitis /; a case report with literature review 2015;1: 16-19.

5. Hunter KM. An infected dentigerous cyst complicated by periostitis ossificans. Case report1955; 273-279.

6. Vijay P, Singhal I, Pardhe N, et al. Case report inflammatory dentigerous cyst /a rare case report. Int J Cur Med \& Pharmac ; 3-5.

7. Peng C, Huang Y, Lu M. Science direct intraosseous verrucous carcinoma arising from an infected dentigerous cyst $\mathrm{d}$ a case report. J Formos Med Assoc 2015;114: 764-768.
8. Reports C, Gonzalez SM, Spalding PM, et al. Case report a dentigerous cyst associated with bilaterally impacted mandibular canines in a girl: a case report $2011 ; 2-5$.

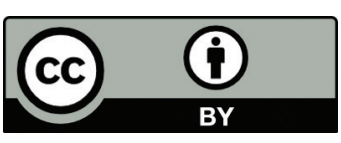

This work is licensed under a Creative Commons Attribution 